\title{
Minireview
}

\section{Chaperonins - keeping a lid on folding proteins}

\author{
Andrew R. Kusmierczyk, Jörg Martin* \\ Department of Molecular Biology, Cell Biology, and Biochemistry, Brown University, P.O. Box G-J2, Providence, RI 02912, USA
}

Received 30 July 2001; revised 8 August 2001; accepted 14 August 2001

First published online 23 August 2001

Edited by Vladimir Skulachev

\begin{abstract}
Two classes of chaperonins are known in all groups of organisms to participate in the folding of newly synthesized proteins. Whereas bacterial type I chaperonins use a reversibly binding cofactor to temporarily sequester folding substrate proteins within the cylindrical chaperonin cavity, type II chaperonins in archaea and the eukaryotic cytosol appear to have evolved a built-in lid for this purpose. Not entirely surprisingly, this has consequences for the folding modes of the two types of chaperonins. (c) 2001 Federation of European Biochemical Societies. Published by Elsevier Science B.V. All rights reserved.
\end{abstract}

Key words: Protein folding; Chaperonin; GroEL; Chaperonin containing TCP-1; Thermosome; GroES

\section{Introduction}

Protein folding in the cell requires the assistance of molecular chaperones [1]. The chaperonins, a sub-group of molecular chaperones found in all domains of life, comprise a family of ATPases of roughly $60 \mathrm{kDa}$ that assemble into twin rings, stacked back-to-back, with a large central cavity where unfolded polypeptides may bind and undergo productive folding (Fig. 1) [2]. Based on their evolutionary origin, chaperonins can be grouped into two distinct classes. Type I chaperonins, found in the bacterial cytosol and in endosymbiontderived organelles of eukaryotes, form heptameric rings and are represented by bacterial GroEL, mitochondrial hsp60, and chloroplast cpn60. Type II chaperonins, present in the eukaryotic cytoplasm and in archaea, include the archaeal thermosome and the eukaryotic CCT (chaperonin containing TCP-1) $[3,4]$. Thermosome rings are eight- or nine-membered, and consist of one to three subunit types $[3,5,6]$. CCT rings consist of eight subunits, each the product of an essential gene [7]. The crystal structures of Escherichia coli GroEL and the thermosome from Thermoplasma acidophilum reveal that all chaperonins share a similar domain architecture [5,8,9]. An equatorial domain forms the interface between individual rings and is the site of ATP binding and hydrolysis, and substrate protein binds at the central face of the apical domain, and the two are connected by a short intermediate domain. GroEL is the best studied chaperonin and its mechanism of action is

*Corresponding author. Fax: (1)-401-863 1201

E-mail address: jorg_martin@brown.edu (J. Martin). understood in some detail (Fig. 1). Our understanding of type II chaperonins currently lags behind that of GroEL, but the emerging picture suggests a degree of similarity in the ATPdriven folding cycle among all chaperonins [10]. However, the two classes of chaperonins also differ from each other in several important respects. In this minireview, we highlight the salient features of chaperonin structure and function while pointing out the known similarities and differences between the two classes (Table 1).

\section{Type I chaperonins - a separate lid for protein folding inside the cylinder cavity}

GroEL, like all type I chaperonins, is assisted in its function by a $10 \mathrm{kDa}$ cochaperonin, GroES, that assembles into a heptamer [11,12]. GroES fulfills two functions: it acts as a lid to seal off the folding chamber and helps displace bound substrate protein into the cavity where it can undergo folding. The nucleotide-dependent interaction between GroEL and GroES has been well characterized. A flexible motif within the cochaperonin subunit, called the mobile loop [13], mediates its binding to a well-defined region in the apical domain of GroEL [9] which also forms part of the hydrophobic surface responsible for binding of substrate proteins [14]. The fact that GroES is essential in vivo [15] supports the view that a sealed cavity is necessary for successful folding of a significant number of substrate proteins to the native state, or at least to a form that is committed to reaching the native conformation. In the absence of GroES, substrate proteins can escape the chaperonin in an unfolded form that is prone to aggregation [16]. Although some model substrate proteins used in in vitro studies can be refolded from GroEL under such conditions, these are proteins that are quite capable of folding spontaneously in bulk solution on their own if released from GroEL without GroES [16]. For these 'non-stringent' substrates, encapsulation in the folding cavity is a moot point, possibly even in vivo.

The GroES-sealed cavity encloses a volume of $175000 \AA^{3}$, sufficiently large to encapsulate a polypeptide of approximately $60 \mathrm{kDa}$ [9]. However, proteins larger than $60 \mathrm{kDa}$ have also been shown to interact with GroEL by coimmunoprecipitation and sodium dodecyl sulfate-polyacrylamide gel electrophoresis [17]. Whether these represent bona fide substrates and, if so, how GroEL deals with them, is not known. The volume of the GroES-sealed cavity is actually twice the volume of the cavity in unliganded GroEL [8,9]. To achieve this increase, GroEL undergoes profound conformational changes, which can be thought of as three rigid body move- 


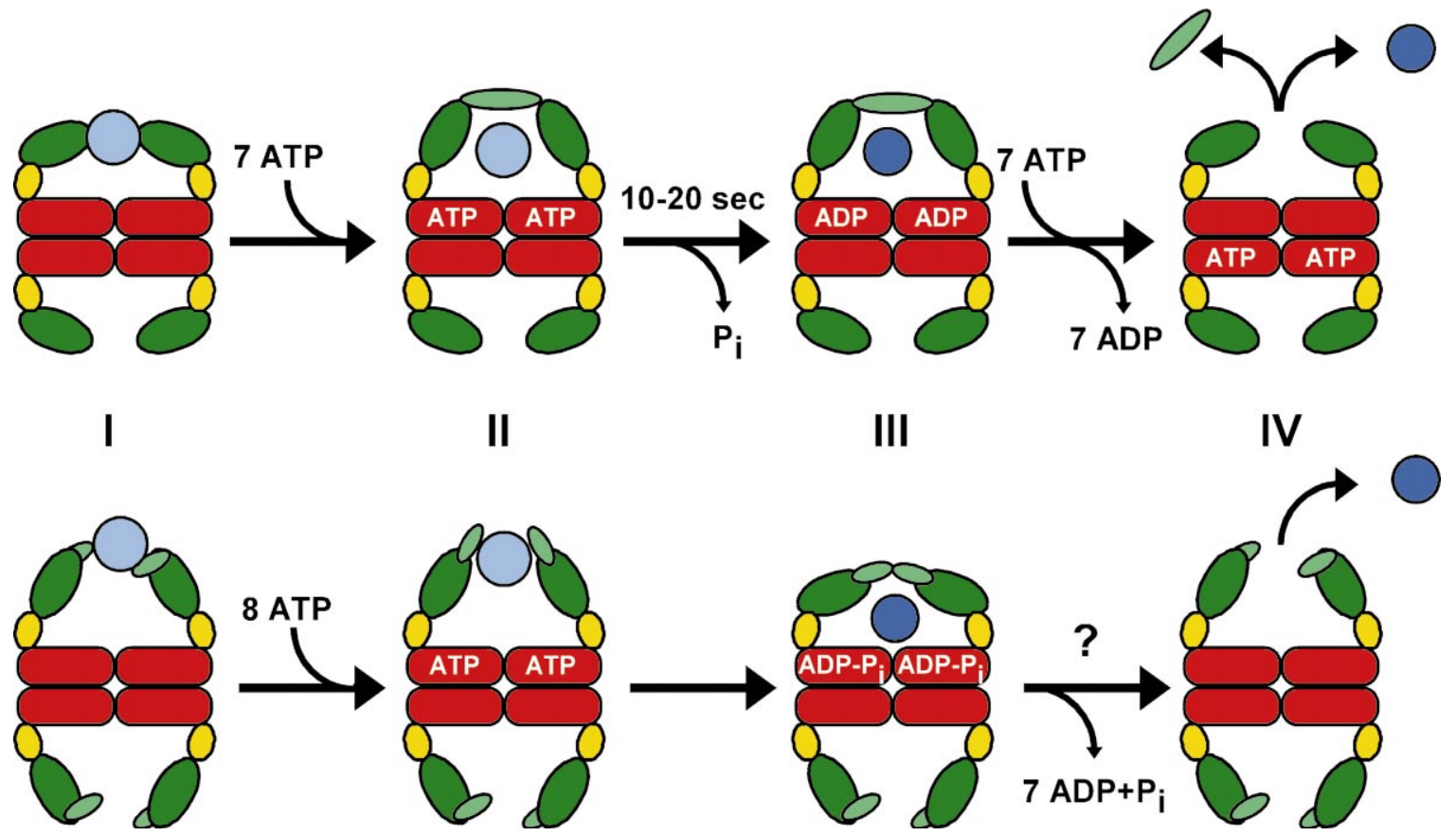

Fig. 1. Chaperonin reaction cycles. Top panel: Type I chaperonins. The binding of substrate protein to one chaperonin ring (I) is followed by the binding of cochaperonin (GroES) and seven molecules of ATP to the same ring (II). The substrate is released into the now-closed cavity and has 10-20 s of folding time before ATP is hydrolyzed (III). Following hydrolysis, the binding of seven molecules of ATP to the opposite ring triggers the dissociation of the cochaperonin and the release of folded substrate protein (IV). Bottom panel: Type II chaperonins. Substrate protein is bound at the apical domains, possibly involving the helical protrusions (I). Binding of eight molecules of ATP then results in rotation of the apical domains to face the cavity (II). Closure of the cavity and release of the bound substrate protein into the interior to fold presumably requires hydrolysis of ATP (III). An - as yet - unknown signal triggers the opening of the chaperonin cavity and release of the folded substrate (IV). The color-coding of the various components is as follows: chaperonin equatorial domain (red), intermediate domain (yellow), and apical domain (dark green); cochaperonin and helical protrusions (light green); unfolded substrate protein (light blue); folded substrate protein (dark blue).

ments occurring about two hinges (Fig. 2). They include: (1) a $25^{\circ}$ downward movement of the intermediate domain which clamps down on the nucleotide binding site of the equatorial domain, (2) a $60^{\circ}$ upward displacement of the apical domain relative to the vertical, and (3) a $90^{\circ}$ clockwise rotation of the apical domain about its longitudinal axis. These domain movements are driven by binding and hydrolysis of ATP, and are essential for the distinct events occurring during the chaperonin reaction cycle.

During such a cycle (Fig. 1), binding of seven molecules of ATP to the substrate-containing ring is followed immediately by the binding of GroES to the same, or cis, ring [18,19]. The bound substrate is thus released into the cavity, which is sealed by the cochaperonin. While inside the cavity, the protein has about 10-20 s to fold [20], after which the seven ATP molecules are hydrolyzed and the GroEL-GroES complex relaxes into a conformation that is ready to dissociate [21]. Binding of seven ATP to the opposite, or trans, ring causes the dissociation of GroES from GroEL, thereby allowing the substrate protein to be released [22]. In the absence of substrate, the rate-limiting step is the aforementioned relaxation of the GroEL-GroES complex, while in the presence of substrate, it is nucleotide hydrolysis itself [21].

Two levels of cooperativity in both binding and hydrolysis of ATP have been observed for GroEL in vitro, and there is emerging evidence that cooperativity is necessary for proper chaperonin function in vivo [23]. First, there exists positive cooperativity within a given ring [24,25]. Positive cooperativity would presumably serve to ensure an all-or-none switch in conformation required to both bind GroES and release substrate protein into the cavity. This is important in light of the fact that substrate proteins interact with several subunits of

Table 1

Features of type I and type II chaperonins

\begin{tabular}{|c|c|c|c|}
\hline \multirow[t]{2}{*}{ Chaperonin features } & \multirow[t]{2}{*}{ Type I chaperonins } & \multicolumn{2}{|l|}{ Type II chaperonins } \\
\hline & & $\overline{\mathrm{CCT}}$ & Thermosome \\
\hline Number of subunits per chaperonin ring & 7 & 8 & 8 or 9 \\
\hline Different subunits per complex & generally $1-2$ & 8 & $1-3$ \\
\hline Lid function & separate cochaperonin (GroES) & \multicolumn{2}{|c|}{ built-in lid } \\
\hline Volume of closed cavity & $175000 \AA^{3}$ & \multicolumn{2}{|c|}{$130000 \AA^{3}$} \\
\hline ATP binding mode & concerted (MWC model) & sequential? (KNF model) & N/A \\
\hline Positive intra-ring cooperativity & yes & yes & N/A \\
\hline Negative inter-ring cooperativity & yes & yes & yes? \\
\hline Folding mode & post-translational & mostly cotranslational & N/A \\
\hline
\end{tabular}


A

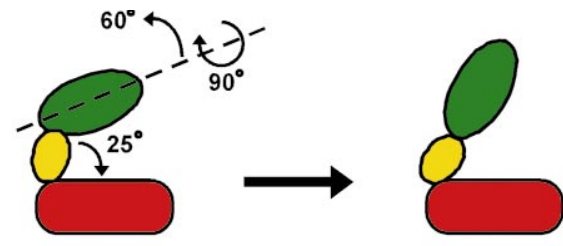

open closed

B

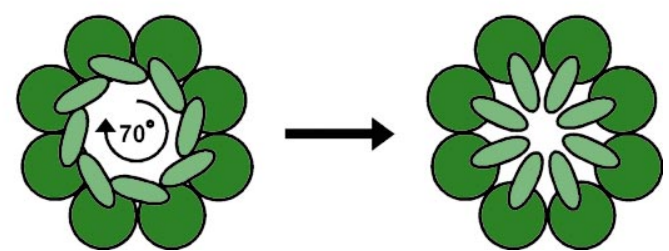

Fig. 2. Domain movements occurring during the open-to-closed transition of the chaperonin reaction cycles. A: Side-view of a type I chaperonin subunit. The downward $25^{\circ}$ rotation of the intermediate domain (yellow) precedes the upward $60^{\circ}$ displacement and $90^{\circ}$ clockwise rotation of the apical domain (green). B: Top-view of the eight apical domains in a type II chaperonin ring. A $70^{\circ}$ clockwise rotation of the apical core domains (dark green) orients the helical protrusions (light green) towards the center of the cavity. To completely seal off the cavity, the apical domains also tilt $30^{\circ}$ downwards (not shown).

GroEL simultaneously [26]. Second, information is conveyed between the two rings in the form of negative cooperativity [25] such that the occupancy of one ring by ATP decreases the affinity of the second ring for ATP. GroEL has been described as a 'two-stroke' motor in which each ring takes turn in being 'folding-active', and it may be that negative cooperativity contributes to this directionality in the chaperonin folding cycle $[27,28]$.

\section{Type II chaperonins - made with a built-in lid}

One important way in which type II chaperonins differ from their type I brethren is in the absence of a partner cochaperonin. So, how do type II chaperonins keep a lid on events occurring inside the folding cavity? The first clue came from the crystal structure of the isolated apical domain of the T. acidophilum thermosome [29]. The structure made it immediately apparent that a helical region present in the thermosome, but absent in GroEL, protruded towards what would be the center of the cavity in an intact chaperonin. This 'helical protrusion' was proposed to act as a built-in lid, and the presence of conserved hydrophobic residues in the protrusion also made it a candidate for the region involved in substrate binding [29]. The crystal structure of the complete thermosome confirmed the prediction that the protrusion could close off the cavity [5]. Interestingly, the structure also showed that a portion of the protrusion, which was helical in the isolated apical domain, now adopted a $\beta$-strand conformation. Taken together, the crystal structures and accompanying electron microscopy [30,31] made it attractive to envision the following model (Fig. 2). In one conformation, the protrusion would be facing away from the cavity, thereby exposing the hydrophobic residues for binding. In another conformation, the protrusion would occlude the cavity, thereby encapsulating the substrate protein and releasing it into the interior to fold. Though the role of the protrusion as built-in lid has gained acceptance, it should be pointed out that its role as substrate-binding site has yet to be demonstrated. Results from cryoelectron microscopy studies of CCT-tubulin and CCT-actin substrate protein complexes indicate that even though tubulin appears bound at the level of the protrusions, actin appears to be bound well below them [32,33].

To isolate a substrate protein in the closed cavity is expected to increase the folding yield because it would eliminate unproductive side reactions, such as aggregation. The volume of the closed cavity in type II chaperonins has been estimated to be approximately $130000 \AA^{3}$, placing a size exclusion limit of about $50 \mathrm{kDa}$ on proteins that can be fully encapsulated [5]. Yet, CCT has been shown to fold larger proteins, such as luciferase [4]. Additionally, a recent finding demonstrated that actin and tubulin could be folded just as efficiently by a CCT in which the cavity was partially occupied by a monoclonal antibody directed against one of the chaperonin subunits [34]. The authors raised the possibility that folding by type II chaperonins may not require complete closure of the cavity. Would this be compatible with the role of CCT in vivo? It is known that CCT is associated with translating ribosomes [35] and that folding of proteins in eukaryotes occurs mostly cotranslationally [36]. CCT has also been shown to interact with nascent chains as they are extruded from the protein synthesis machinery, and to participate in their folding to the native state $[35,37]$. CCT need not accept the substrate protein directly from the ribosome, as nascent chains can interact with other chaperones first (such as hsp70 and prefoldin) before being delivered to the chaperonin [35,38]. If a polypeptide chain is being continuously fed from the ribosome, to which it is still attached via its C-terminus, one could envision the need to keep the cavity open in order to accommodate the growing protein. A detachable lid, like GroES, would then interfere with the threading of polypeptide to the chaperonin.

The rate of ATP turnover by CCT in vitro is anemic compared to GroEL (0.0119-0.0183 s ${ }^{-1}$ vs. $0.27-0.40 \mathrm{~s}^{-1}$ for single and double rings, respectively) [39]. However, the presence of denatured substrate protein has been shown to increase this rate up to eight-fold [40], leading to a CCT single-ring turnover time of $70 \mathrm{~s}$. A current model holds that de novo protein folding in eukaryotes proceeds in a domain-wise manner, with contiguous domains folding individually as they emerge from the ribosome (reviewed in [41]). Given the eukaryotic translation rate of approximately five amino acids per second, a $150-300$ amino acid domain would take $30-60$ s to be synthesized, well within the $70 \mathrm{~s}$ window of opportunity for one turn of the chaperonin cycle. CCT could then cycle between open and (partially) closed states, thereby gobbling up successive stretches of polypeptide in a 'pac-man'-like fashion as they come off the ribosome. While CCT may thus be very well suited to cotranslational folding, bacterial GroEL is not recruited to translating ribosomes and has been demonstrated to act post-translationally [42]. A detachable lid is therefore of no concern with regards to interference during bacterial translation. Furthermore, the complete closure of the cavity by GroES is actually beneficial given that bacterial translation proceeds at a much more rapid pace (about 10 times faster than in eukaryotes), and protein folding is thought to be largely, although not necessarily exclusively, post-translational $[36,41]$. Without the added security that the tethering of a folding nascent protein to the ribosome provides in eukary- 
otes, it may become more beneficial to prevent premature escape of an unfolded protein by completely sealing off the GroEL cavity.

\section{Chaperonins and nucleotides $C$ open or closed?}

As mentioned above, the crystal structure of the thermosome shows the chaperonin in a fully closed conformation, with the helical protrusions in both rings pointing towards the center of the cavity [5]. What stage of the chaperonin cycle does this structure actually represent? Cryoelectron tomography and microscopy of nucleotide-free type II chaperonins reveal them to be in an open conformation with the helical protrusions contacting the apical domains of neighboring subunits, thereby giving substrate protein full access to the central cavity in both rings [30]. The ATP-bound form of CCT is even slightly more open than the nucleotide-free form $[31,43]$. In contrast to a nucleotide-free CCT, the apical domains in an ATP-bound ring are rotated $70^{\circ}$ clockwise such that their tips now face the central cavity, and the equatorial domain moves out slightly (Fig. 2). Although the helical protrusions were not resolved in structural data of ATP-bound CCT [31], they remain sensitive to proteolysis [44], indicating that ATP hydrolysis is required to drive the chaperonin into the fully closed state where the protrusions are protected and fully cover the cavity. There is evidence to suggest that in the absence of substrate protein, the rate-limiting step of the thermosome ATPase activity is a slow, post-hydrolysis isomerization into a closed ADP- $P_{\mathrm{i}}$ state prior to phosphate release $[43,45]$. Whether this is also the case in the presence of substrate protein is not known. In order to progress into this fully closed form, the apical domains would have to tilt $30^{\circ}$ downward [46]. Therefore, the thermosome rings in the crystal structure likely represent the chaperonin in an ADP- $P_{\mathrm{i}}$ conformation. Another conformation, one in which one ring is open and the other is closed, has been recently observed for thermosomes from two different species of archaea [46,47]. This asymmetrical, bullet-shaped conformation is highly reminiscent of the asymmetrical GroES-bound GroEL complex.

Our understanding of the ATPase cycle of type II chaperonins (Fig. 1) is not as detailed yet as that of GroEL. Despite the fact that the greatest degree of sequence similarity among all chaperonins lies in the equatorial domain, which contains the nucleotide binding pocket, differences between the two classes have begun to emerge. For example, the chaperonins differ in their monovalent cation requirements for ATP hydrolysis [2,10]. Analogous to GroEL, two levels of cooperativity have recently been reported for CCT [39]. However, the value of the Hill coefficient for the positive cooperativity existing within each ring, was lower for CCT than for GroEL under identical conditions. The authors suggest that this might be due to the different affinities of the eight distinct CCT subunits for ATP [39]. It has been proposed that a hierarchy exists among the CCT subunits in terms of ATP binding [48] which correlates well with the specific order in which the subunits are arranged within a ring [49]. Such a hierarchy implies that the binding of ATP to CCT, and the conformational changes that accompany it, may be sequential (KNF model of cooperativity) rather than all-or-none as proposed for GroEL. In contrast, the negative cooperativity that was found to exist between the two CCT rings was considerably stronger than that which exists in type I chaperonins [39].
Furthermore, unlike in GroEL, the binding of ATP to the second ring appears not to decrease the maximal ATPase activity of CCT [39]; the ATPase activity nearly doubles instead. It would appear that the nature of the inter-ring communication may not be the same for type I and II chaperonins. Perhaps this is not surprising given the different quaternary arrangement of the subunits between the two classes. Whereas the subunits between two rings in type I chaperonins are staggered, they are aligned in register in type II chaperonins $[5,8,9]$.

A wealth of information concerning chaperonin function has emerged over the past decade, with GroEL at the forefront. To complete the picture, the spotlight has begun to shift to type II chaperonins. Based on current data, the emerging synthesis of type I and type II chaperonin function will likely highlight two ingenious, albeit different solutions that have evolved to arrive at efficient protein folding inside a cylindrical cavity.

Acknowledgements: The authors thank Jennifer Carr for critically reading the manuscript. Work in the authors' laboratory was supported by National Institutes of Health Grant GM54534.

\section{References}

[1] Frydman, J. (2001) Annu. Rev. Biochem. 70, 603-647.

[2] Ranson, N.A., White, H.E. and Saibil, H.R. (1998) Biochem. J. $333,233-242$

[3] Trent, J.D., Nimmesgern, E., Wall, J.S., Hartl, F.U. and Horwich, A.L. (1991) Nature 354, 490-493.

[4] Frydman, J., Nimmesgern, E., Erdjument-Bromage, H., Wall, J.S., Tempst, P. and Hartl, F.U. (1992) EMBO J. 11, 47674778.

[5] Ditzel, L., Löwe, J., Stock, D., Stetter, K.-O., Huber, H., Huber, R. and Steinbacher, S. (1998) Cell 93, 125-138.

[6] Archibald, J.M., Logsdon, J.M. and Doolittle, W.F. (1999) Curr. Biol. 9, 1053-1056.

[7] Kubota, H., Yokota, S., Yanagi, H. and Yura, T. (1999) Eur. J. Biochem. 262, 492-500.

[8] Braig, K., Otwinowski, Z., Hegde, R., Boisvert, D.C., Joachimiak, A., Horwich, A.L. and Sigler, P.B. (1994) Nature $371,578-586$

[9] Xu, Z., Horwich, A.L. and Sigler, P.B. (1997) Nature 388, 741750 .

[10] Gutsche, I., Essen, L.-O. and Baumeister, W. (1999) J. Mol. Biol. 293, 295-312

[11] Chandrasekhar, G.N., Tilly, K., Woolford, C., Hendrix, R. and Georgopoulos, C. (1986) J. Biol. Chem. 261, 12414-12419.

[12] Hunt, J.F., Weaver, A.J., Landry, S.J., Gierasch, L. and Deisenhofer, J. (1996) Nature 379, 37-45.

[13] Landry, S.J., Zeilstra-Ryalls, J., Fayet, O., Georgopoulos, C. and Gierasch, L.M. (1993) Nature 364, 255-258.

[14] Fenton, W.A., Kashi, Y., Furtak, K. and Horwich, A.L. (1994) Nature 371, 614-619.

[15] Fayet, O., Ziegelhoffer, T. and Georgopoulos, C. (1989) J. Bacteriol. 171, 1379-1385.

[16] Martin, J., Langer, T., Boteva, R., Schramel, A., Horwich, A.L. and Hartl, F.U. (1991) Nature 352, 36-42.

[17] Ewalt, K.L., Hendrick, J.P., Houry, W.A. and Hartl, F.U. (1997) Cell 90, 491-500.

[18] Langer, T., Pfeifer, G., Martin, J., Baumeister, W. and Hartl, F.U. (1992) EMBO J. 11, 4757-4765.

[19] Martin, J., Mayhew, M., Langer, T. and Hartl, F.U. (1993) Nature 366, 228-233.

[20] Burston, S.G., Ranson, N.A. and Clarke, A.R. (1995) J. Mol. Biol. 249, 138-152.

[21] Rye, H.S., Roseman, A.M., Chen, S., Furtak, K., Fenton, W.A. Saibil, H.R. and Horwich, A.L. (1999) Cell 97, 325-338.

[22] Rye, H.S., Burston, S.G., Fenton, W.A., Beechem, J.M., Xu, Z., Sigler, P. and Horwich, A.L. (1997) Nature 388, 792-798 
[23] Fridmann, Y., Ulitzur, S. and Horovitz, A. (2000) J. Biol. Chem. 275, 37951-37956.

[24] Jackson, S.G., Staniforth, R.A., Halsall, D.J., Atkinson, T., Holbrook, J.J., Clarke, A.R. and Burston, S.G. (1993) Biochemistry 32, 2554-2563.

[25] Yifrach, O. and Horovitz, A. (1995) Biochemistry 34, 5303-5308.

[26] Farr, G.W., Furtak, K., Rowland, M.B., Ranson, N.A., Saibil, H.R., Kirchhausen, T. and Horwich, A.L. (2000) Cell 100, 561573.

[27] Kad, N.M., Ranson, N.A., Cliff, M.J. and Clarke, A.R. (1998) J. Mol. Biol. 278, 267-278.

[28] Yifrach, O. and Horovitz, A. (2000) Proc. Natl. Acad. Sci. USA 97, 1521-1524.

[29] Klumpp, M., Baumeister, W. and Essen, L.-O. (1997) Cell 91, 263-270.

[30] Nitsch, M., Walz, J., Typke, D., Klumpp, M., Essen, L.-O. and Baumeister, W. (1998) Nat. Struct. Biol. 5, 855-857.

[31] Llorca, O., Smyth, M.G., Carrascosa, J.L., Willison, K.R., Radermacher, M., Steinbacher, S. and Valpuesta, J.M. (1998) Nat. Struct. Biol. 6, 639-642.

[32] Llorca, O., McCormack, E., Hynes, G.M., Grantham, J., Cordell, J., Carrascosa, J.L., Willison, K.R., Fernández, J.J. and Valpuesta, J.M. (1999) Nature 402, 693-696.

[33] Llorca, O., Benito-Martín, J., Ritco-Vonsovici, M., Grantham, J., Hynes, G.M., Willison, K.R., Carrascosa, J.L. and Valpuesta, J.M. (2000) EMBO J. 19, 5971-5979.

[34] Grantham, J., Llorca, O., Valpuesta, J.M. and Willison, K.R. (2000) J. Biol. Chem. 275, 4587-4591.
[35] Frydman, J., Nimmesgern, E., Ohtsuka, K. and Hartl, F.U. (1994) Nature 370, 111-117.

[36] Netzer, W. and Hartl, F.U. (1997) Nature 388, 343-349.

[37] McCallum, C.D., Do, H., Johnson, A.E. and Frydman, J. (2000) J. Cell Biol. 149, 591-601.

[38] Hansen, W.J., Cowan, N.J. and Welch, W.J. (1999) J. Cell Biol. $145,265-277$.

[39] Kafri, G., Willison, K.R. and Horovitz, A. (2001) Protein Sci. 10, 445-449.

[40] Melki, R., Rommelaere, H., Leguy, R., Vandekerckhove, J. and Ampe, C. (1996) Biochemistry 35, 10422-10435.

[41] Netzer, W.J. and Hartl, F.U. (1998) Trends Biochem. Sci. 23, 6873.

[42] Gaitanaris, G.A., Vysokanov, A., Hung, S.Z., Gottesman, M. and Gragerov, A. (1994) Mol. Microbiol. 14, 861-869.

[43] Gutsche, I., Holzinger, J., Rößle, M., Heumann, H., Baumeister, W. and May, R.P. (2000) Curr. Biol. 10, 405-408.

[44] Szpikowska, B.K., Swiderek, K.M., Sherman, M.A. and Mas, M.T. (1998) Protein Sci. 7, 1524-1530.

[45] Gutsche, I., Mihalache, O. and Baumeister, W. (2000) J. Mol. Biol. 300, 187-196.

[46] Schoehn, G., Quaite-Randall, E., Jiménez, J.L., Joachimiak, A. and Saibil, H.R. (2000) J. Mol. Biol. 296, 813-819.

[47] Schoehn, G., Hayes, M., Cliff, M., Clarke, A.R. and Saibil, H.R. (2000) J. Mol. Biol. 301, 323-332.

[48] Lin, P. and Sherman, F. (1997) Proc. Natl. Acad. Sci. USA 94, 10780-10785.

[49] Liou, A.K.F. and Willison, K.R. (1997) EMBO J. 16, 4311-4316. 